Cahiers de recherches médiévales

\title{
Théorie et définition des poisons à la fin du Moyen Âge
}

Joël Chandelier

\section{(2) OpenEdition}

1 Journals

Édition électronique

URL : https://journals.openedition.org/crm/11500

DOI : $10.4000 / \mathrm{crm} .11500$

ISSN : 1955-2424

Éditeur

Honoré Champion

\section{Édition imprimée}

Date de publication : 15 juin 2009

Pagination : 23-38

ISSN : $1272-9752$

\section{Référence électronique}

Joël Chandelier, "Théorie et défınition des poisons à la fin du Moyen Âge », Cahiers de recherches médiévales [En ligne], 17 | 2009, mis en ligne le 15 juin 2012, consulté le 15 décembre 2022. URL : http://journals.openedition.org/crm/11500 ; DOI : https://doi.org/10.4000/crm.11500

Ce document a été généré automatiquement le 15 décembre 2022.

Tous droits réservés 


\title{
Théorie et définition des poisons à la fin du Moyen Âge
}

\author{
Joël Chandelier
}

1 Depuis quelques années, l'histoire des poisons et l'analyse de leur usage dans la société ont connu un intérêt renouvelé1. L'objectif de cette étude est quelque peu différent de celui qui a largement prévalu jusqu'ici : il s'agira pour nous de comprendre non pas quels étaient les poisons employés et l'utilisation qui en était faite au Moyen Âge, mais quel fut le discours scientifique des médecins à leur sujet, et plus spécifiquement quelle fut la théorie toxicologique adoptée par les savants médiévaux pour expliquer le fonctionnement du venin dans le corps humain. En effet, la plupart des traités sur le sujet publiés à la fin du Moyen Âge, souvent simplement intitulés De venenis, présentent, avant la liste des diverses substances et de leurs effets, un exposé théorique visant à analyser le mode d'action du produit. Cette partie des traités n'a que rarement été étudiée et, si ce n'est dans quelques travaux anciens, le sujet n'a jusqu'ici guère retenu l'attention'. Pourtant, la publication de l'article fondamental d'Alain Touwaide sur la théorie toxicologique de Galien invite à reprendre ce dossier : son travail montre que non seulement cette toxicologie présentait un caractère synthétique et original, mais également qu'il faut peut-être y voir, au moins en partie, l'origine même de la théorie pharmacologique de l'auteur ${ }^{3}$. Notre projet est donc de comprendre comment cette présentation a été adoptée, et avec quelles évolutions, au cours des derniers siècles du Moyen Âge.

$2 \mathrm{Au}$ point de départ des débats antiques et médiévaux sur les venins, une question simple : quelle est la différence entre un poison et un médicament? Y a-t-il entre les deux genres de substance une distinction de nature, ou simplement d'intensité et d'effet? Si le problème n'était presque jamais posé en ces termes, c'est là que se trouvait à l'évidence la difficulté principale. En effet, les premiers textes traitant de la question, comme les ouvrages perdus de Mithridate ou la Matière médicale de Dioscoride, paraissent avoir eu une approche essentiellement empirique, énumérant simplement l'action des substances et les antidotes possibles ${ }^{4}$. Pour cette raison, la spécificité du poison était mise en avant : l'objectif du médecin était de déterminer la 
nature particulière de chaque venin, afin de prendre les contre-mesures appropriées à celui-ci, et à lui seul ${ }^{5}$. Si cette attitude avait l'avantage d'être immédiatement utile, en donnant au médecin les moyens de déterminer, à partir de l'analyse de symptômes connus, la nature de la substance et l'antidote à administrer, elle ne résolvait pas la question du mode d'action du poison dans le corps humain. Pour cette raison, le statut même du venin restait problématique et ambigu, comme l'atteste le terme grec employé, pharmakon, qui pouvait tout aussi bien désigner le médicament que le poison ${ }^{6}$, tandis qu'à l'inverse les empoisonnements étaient parfois liés à la magie et aux maléfices, sortant alors en partie du champ de la médecine proprement dite ${ }^{7}$.

3 La multiplication des traités sur les venins rédigés par des praticiens dans le monde arabe comme en Occident à partir de la fin du XIII ${ }^{e}$ siècle suggère une tentative d'intégration à la médecine savante de cette matière ambiguë et complexe. Notre objet sera d'observer et d'analyser ce mouvement, qui fait rentrer dans le cadre du savoir scolastique un domaine jusqu'alors situé à la frontière de la science ${ }^{8}$. Pour cela, nous présenterons tout d'abord la réponse de Galien, pour ensuite étudier les apports arabes et leur intégration en Occident.

\section{Médicaments et poisons chez Galien}

4 Comme souvent, Galien s'appuie dans le cas des poisons sur la littérature antérieure, qu'il rassemble en une grande et originale synthèse. Selon une conception répandue, chaque venin était considéré comme une substance particulière, liée à un remède unique et précis. Il ne semble pas qu'il y ait alors eu, à proprement parler, de théorie globale: chaque substance était traitée de manière individualisée, sans véritable recherche d'une définition commune de l'essence du poison. Le défi de Galien était donc d'intégrer les informations sur les substances issues de cette tradition empirique dans un cadre théorique plus complet ${ }^{9}$.

5 Pour répondre à la question de l'explication du mode de fonctionnement dans le corps des substances venimeuses, Galien décide d'intégrer complètement la toxicologie à sa théorie pharmacologique. Pour lui, les poisons agissent, comme les médicaments simples, par leur complexion, en étant chauds, froids, humides ou secs. Galien introduit toutefois une distinction : certains venins agissent par leur nature propre, c'est-à-dire qu'ils nuisent systématiquement au corps humain, tandis que d'autres, comme la laitue, ne le font que s'ils sont pris en quantité excessive - dans le cas contraire, les poisons de ce type peuvent avoir un effet nul, voire positif ${ }^{10}$. Pour Galien, le poison a donc une action avant tout humorale, qui ne se distingue pas réellement de celle des médicaments, ni même, pour ses effets, des maladies en général. Une telle conception a d'importantes conséquences. En premier lieu, l'action des venins ne peut, pour cette raison, être distinguée des maladies d'humeur identique : en principe, il est impossible de différencier le déséquilibre humoral provoqué par un poison de celui relevé dans le cas d'une maladie du même type ${ }^{11}$. De ce fait, le traitement à apporter aux poisons n'est pas différent de celui des maladies, ce qui autorise Galien à rapprocher, par exemple, l'effet du poison de l'action de la peste, tous deux agissant sur le corps tout entier ${ }^{12}$.

On voit donc que le médecin de Pergame intègre totalement le poison à sa pharmaceutique, et même à l'ensemble de sa théorie humorale. Le poison n'a, en fin de compte, aucun caractère particulier. Il s'agit en réalité d'une médecine, mais dont l'action est négative pour le corps humain, soit par sa qualité propre, soit par la 
quantité ingérée. Si cette présentation de la toxicologie a l'intérêt d'être cohérente avec l'ensemble de la médecine galénique, elle pose toutefois un certain nombre de problèmes. En effet, si le poison a toutes les caractéristiques humorales d'un médicament, comment le distinguer de celui-ci? De même, si le venin administré provoque des effets semblables à ceux d'une maladie, comment le médecin peut-il établir un diagnostic et, partant, fournir au patient l'antidote approprié, s'il ne connaît pas d'avance la substance ingérée ? Ces questions n'ont pas été posées directement par Galien, mais sa volonté d'intégrer les venins à la matière médicale rendait délicate toute réponse. Le problème restant donc incomplètement résolu, les auteurs successifs s'attachèrent logiquement à préciser et améliorer la construction du maître de l'Antiquité.

\section{L'apport d'Avicenne : l'introduction de la forme spécifique}

7 La théorie toxicologique de Galien était présentée de manière éparse dans ses divers ouvrages, sans faire l'objet d'une véritable présentation cohérente; les premiers ouvrages encyclopédiques arabes conservèrent cette attitude ${ }^{13}$. Dans le Liber ad Almansorem de Rāzī et dans la Regalis dispositio (ou Pantegni) d'al-Mağūsī (Haly Abbas), rédigés aux $\mathrm{IX}^{\mathrm{e}}$ et $\mathrm{X}^{\mathrm{e}}$ siècles, la part occupée par la théorie des poisons est fort réduite ${ }^{14}$. Le premier des deux ouvrages consacre certes un livre entier aux venins (le huitième), mais il ne comporte pas à proprement parler de description théorique de l'action des poisons, le texte se consacrant dès le premier chapitre aux remèdes à utiliser ${ }^{15}$. Quant à al-Mağūsī, il choisit de ne placer la doctrine des poisons dans aucun livre particulier, préférant la répartir dans des chapitres situés en plusieurs endroits de son œuvre. En réalité, le premier à réserver une place particulière aux poisons dans l'ensemble de sa théorie médicale est Avicenne. Dans son Canon, une fen (partie) entière du livre IV est exclusivement consacrée aux venins, à leur action, à leur description et aux remèdes que l'on peut y apporter ${ }^{16}$. Sa présentation est marquée par l'apparition d'une nouveauté conceptuelle, sur laquelle il faut s'attarder : la forme spécifique ${ }^{17}$.

Le concept de forme spécifique est, au départ, utilisé par Avicenne dans le cadre de la pharmacologie. Dans le chapitre sur l'alimentation et la boisson, il affirme que tout substance ingérée peut agir selon trois modes : par sa seule qualité, par sa matière, ou par sa forme spécifique ${ }^{18}$. Le premier cas correspond par exemple à la médecine chaude, qui échauffe le corps, ou à la médecine froide, qui le refroidit; dans ces cas, la médecine agit sur le corps sans être matériellement assimilée à lui. Le second mode d'action est celui de la nourriture ; sa matière est assimilée, en ayant une influence, plus réduite toutefois que celle des médecines, par sa complexion propre qui modifie celle du corps. L'action par la forme spécifique est quant à elle fort différente; la complexion de la médecine n'entre plus en ligne de compte, puisque cette forme spécifique est une propriété unique et particulière d'une médecine ou d'un aliment donné. Avicenne déclare ainsi :

C'est une forme qu'acquiert une substance postérieurement à la complexion; elle dérive du mélange des éléments simples qui la caractérisent. De ce mélange résulte une aptitude à recevoir une espèce, une forme ajoutée à celle que les simples possèdent (...). C'est une sorte de perfection acquise par la matière en fonction de cette aptitude dérivée de la complexion; il en est ainsi de la vertu attractive de l'aimant ${ }^{19}$. 
Dans le chapitre consacré à la confection de la thériaque, Avicenne précisait l'origine de la forme spécifique, en la liant à une sorte de fermentation ${ }^{20}$. L'originalité de la théorie avicennienne réside bien sûr dans l'introduction du concept de cette forme spécifique, que l'on ne trouvait pas dans une pharmacologie galénique qui se limitait aux deux autres modes d'action, même si le terme et l'idée sont tirés de certains passages peu clairs de ce même Galien, parlant de tota substantia ${ }^{21}$. L'innovation répondait à un besoin. En effet, il apparaissait souvent difficile de justifier, par la seule théorie des qualités de chaud, froid, humide et sec, l'action de certains médicaments, et la forme spécifique apportait une souplesse bienvenue à une théorie galénique qui, poussée à l'extrême, pouvait se révéler difficilement applicable, notamment dans les cas très particuliers d'un médicament précis soignant une maladie spécifique ne s'expliquant pas par la théorie des humeurs.

10 Mais la théorie de la forme spécifique portait en elle une autre conséquence, d'une très grande portée: puisque chaque forme substantielle était spécifique à chaque médicament composé, et ne dépendait pas uniquement des éléments qui le compose, il était impossible d'en connaître les propriétés par le raisonnement, c'est-à-dire en étant attentif au goût, à la couleur, et à d'autres caractéristiques de la substance que les médecins avaient appris à relier aux qualités fondamentales de chaud, d'humide, de froid et de sec. De par leur caractère unique, qui n'était ni théorisable ni généralisable, les propriétés dues à la forme spécifique ne pouvaient donc être connues que par l'expérience.

11 L'action par la forme spécifique pouvait facilement s'utiliser pour expliquer l'effet des venins: l'une des citations de Galien à l'appui de laquelle Avicenne introduisait ce concept n'était-elle du reste pas tirée d'un passage où le maître grec évoquait l'action des poisons ? ${ }^{22}$ Dans les cinq premiers chapitres de la fen 6 du livre IV est donc affirmée une distinction majeure. Comme le déclare le médecin et philosophe persan, en exergue du chapitre 2 Sermo universalis de venenis que bibuntur, «il y a deux espèces de venin: ceux faisant leur opération par la qualité qui se trouve en eux, et ceux l'effectuant par leur forme et toute leur substance $\aleph^{23}$. Avicenne précise ensuite l'action concrète de ces divers venins. Pour ceux procédant par la complexion, il reprend le concept galénique des qualités secondes, qui viennent s'ajouter à la qualité première (chaud, froid, humide ou sec): le poison peut ainsi être corrosif, putréfiant, inflammant, refroidissant, ou bloquant les voies respiratoires ${ }^{24}$. Dans le cas de ceux agissant par la forme spécifique, la distinction se fait en revanche entre ceux agissant sur un membre particulier ou sur le corps tout entier, comme l'opium ${ }^{25}$. Le médecin est donc capable, d'après les symptômes du patient, de distinguer deux genres de venins: tandis que l'action par la complexion se signale par l'effet de la qualité seconde, par exemple l'inflammation, la corrosion ou encore l'échauffement du corps, l'intervention de la forme spécifique peut être identifiée quand «il n'apparait qu'un affaiblissement des vertus, une sueur froide et une syncope »; ce sont, dans ce cas, les pires venins ${ }^{26}$.

Bien sûr, l'introduction par Avicenne de la forme spécifique dans la théorie toxicologique n'est pas sans conséquence sur la manière dont doivent être considérés le poison et les remèdes à y apporter. L'idée que certains venins s'opposent par toute leur substance au corps humain permet à Avicenne de mieux les définir. En effet, puisque leur action n'est plus uniquement humorale, il devient possible au médecin d'en déterminer les symptômes, et donc de mieux choisir les antidotes spécifiques, qui sont ceux empêchant les substances toxiques d'atteindre le cœur, comme la thériaque ${ }^{27}$ : la 
nouvelle présentation tend donc à mieux distinguer, du point de vue de la thérapeutique, les empoisonnements des simples maladies. En revanche, les nouveautés introduites par le médecin persan ne remettent pas en cause, bien au contraire, le lien fort que nous avions observé chez Galien entre médecine et poison : tous deux ont une action identique, par la complexion ou la forme spécifique, et le poison reste, en somme, un médicament dont l'effet est mauvais pour le corps humain.

Le succès de la théorie d'Avicenne fut, semble-t-il, immédiat dans le monde arabe, de même que celui du concept nouveau de forme spécifique. Le De venenis d'Averroès (m. 1198), commence par exemple ainsi : «Toutes les espèces de mortifères se tiennent en deux genres, dans lesquels les espèces sont pratiquement infinies. Le premier est celui de ceux qui tuent par la forme spécifique (...). Le second genre est celui de ceux qui tuent par la forme de la complexion, c'est-à-dire par leurs qualités premières ${ }^{28}$. L'emploi de la forme spécifique, parallèlement à son usage intensif dans le cadre de la pharmacologie, paraît donc avoir été adopté sans hésitation par la toxicologie arabe.

\section{La théorie toxicologique chez les Latins : le De venenis de Cristoforo degli Onesti}

14 Le succès de la synthèse toxicologique avicennienne, largement attesté chez les auteurs arabes $\mathrm{du} \mathrm{XII}^{\mathrm{e}}$ siècle, ne se dément pas dans le monde latin: du reste, les auteurs occidentaux sont, de par les traductions effectuées à partir du XII ${ }^{e}$ siècle, largement dépendants de la reformulation arabe du galénisme. Le Canon, traduit par Gérard de Crémone dans la seconde moitié du XII ${ }^{\mathrm{e}}$ siècle, commence à avoir une diffusion massive à partir du milieu du siècle suivant ${ }^{29}$. Quant au traité De venenis d'Averroès, il est mis en latin peu avant 1300, sans doute par Armengaud Blaise, traducteur montpelliérain de la fin du XIII ${ }^{e}$ et du début du XIV ${ }^{e}$ siècle. Ces ouvrages, ainsi que les autres synthèses traduites à la même époque, contribuent à encourager la rédaction de traités particulier sur les poisons, qui se multiplient à partir de la fin du XIII ${ }^{e}$ siècle ${ }^{30}$. L'un des premiers textes à avoir été écrits sur le sujet, et sans doute celui qui a connu le plus grand succès, est le De venenis de Pietro d'Abano ${ }^{31}$. Nous ne voulons pas revenir en détail sur cet ouvrage bien connu, mais plusieurs éléments doivent être soulignés pour comprendre son rôle et les infléchissements qu'il fait subir à la doctrine toxicologique héritée des Grecs et des Arabes.

Dans l'introduction théorique de son ouvrage, Pietro d'Abano intègre complètement la présentation avicennienne : pour lui, on peut « considérer le venin selon la différence spécifique qui fait différer une espèce de l'autre, et selon ce critère tous les médecins et les philosophes naturels ont dit qu'il y a des venins qui accomplissent leur opération par leur qualité ou complexion, et d'autres qui le font par la vertu dérivant de toute leur espèce, que les médecins appellent forme spécifique $»^{32}$. Toutefois, il y ajoute un élément important. En effet, à la définition purement physique (une fermentation) de la forme spécifique dans le Canon, venait s'en ajouter une autre, tirée du livre Des simples (ou De consolatione medicinarum) du Pseudo-Mésué, et tendant à attribuer son origine à une influence céleste. Pour le Pseudo-Mésué, «tout [simple] est doté d'une double vertu, élémentaire et céleste, la première générale, l'autre propre $\aleph^{33}$. Cette notion ayant été largement adoptée par les médecins dans le cadre de la pharmacologie (on la retrouve chez des contemporains de Pietro d'Abano comme Taddeo Alderotti, professeur à Bologne mort en 1295), Pietro, fort logiquement, insiste sur ce point dans 
son traité des poisons : «[la forme spécifique] n'est rien d'autre que le bénéfice que chaque substance composée à partir des quatre éléments selon une proportion plus ou moins grande de ces éléments obtient, et dont elle bénéficie grâce à l'influence des étoiles fixes qui veillent sur les espèces des composés inférieurs $»^{34}$. L'intégration d'éléments tirés de l'astrologie dans le cadre de la théorie toxicologique ne doit pas nous étonner chez Pietro d'Abano: on sait que l'une de ses préoccupations fut justement de promouvoir son usage auprès des médecins $\mathrm{s}^{35}$. Dans ce cas précis, elle se limitait à une cause lointaine, difficilement accessible au médecin, et, pour cette raison, son adoption ne soulevait aucun problème particulier.

Ainsi présentée, la doctrine toxicologique élaborée au cours des siècles est reprise par la majorité des auteurs du XIV siècle. Francesco da Siena, par exemple, affirme dans son traité des poisons adressé au patriarche de Jérusalem Philippe d'Alençon en 1375 que l'essentiel de ses informations provient d'Avicenne ${ }^{36}$. De même, Gentile da Foligno, auteur prolixe mort en 1348, choisit de ne pas rédiger de traité particulier sur le sujet et se contente de commenter la fen 6 du livre IV du Canon, commentaire dont le texte est conservé dans un manuscrit padouan du milieu du XVe siècle et est imprimé à plusieurs reprises à la Renaissance. L'ensemble de ces traités adopte alors, sans véritable discussion, la division proposée par Avicenne.

Toutefois, il serait faux de dire que ces ouvrages sur les poisons se ressemblent tous. Au contraire, l'un d'entre eux va connaître un succès important, au point de devenir, comme l'affirme Lynn Thorndike, le modèle de tous les traités du XV $\mathrm{XV}^{\mathrm{e}}$ siècle ${ }^{37}$ : il s'agit du De venenis de Cristoforo degli Onesti (Christophorus de Honestis), médecin florentin mort en $1392^{38}$. Peu de choses sont connues sur la vie de ce médecin, qui fut semble-t-il l'élève de Tommaso del Garbo avant d'enseigner à Bologne et Florence dans les décennies 1370 et 1380, et auquel sont attribués, en particulier, un commentaire sur le Tegni de Galien, une exposition à l'Antidotaire de Mésué et une autre sur les deux premières fen du premier livre du Canon d'Avicenne ${ }^{39}$. Son succès, attesté par les utilisations très fréquentes qu'en font, au XV siècle, Sante Ardoino de Pesaro dans son Opus de venenis et Giovanni Martini de'Ferrari de Parme dans son De venenis evitandis et eorum remediis libellus ${ }^{40}$, explique que nous en conservions aujourd'hui plusieurs manuscrits ${ }^{41}$. Nous voulons donc, pour terminer ce rapide survol de la théorie toxicologique médiévale, nous attarder quelque peu sur cet ouvrage et sur ses originalités.

Suivant une tradition désormais bien établie, le traité de Cristoforo degli Onesti se divise en deux grandes parties, la première réunissant cinq questions d'ordre théorique, la seconde présentant, de manière ordonnée, les différents venins et leurs antidotes. Dans le prologue qui introduit le traité, Cristoforo présente un historique de la science médicale, allant d'Apollon, dieu de la médecine, à Averroès et Maïmonide, en passant par les principaux auteurs grecs, byzantins et arabes, l'auteur inscrivant clairement son De venenis dans cette longue filiation ${ }^{42}$. Sont ensuite présentés plusieurs problemata théoriques, portant notamment sur l'action des venins sur le cœur, sur les liens entre peste et poisons ou encore sur l'effet de la thériaque comme antidote universel. Puis, avant de présenter les venins un par un, l'auteur s'attarde sur le classement que l'on peut effectuer des différentes substances ${ }^{43}$.

19 Cristoforo degli Onesti distingue cinq manières de classer les poisons. La première consiste à séparer les venins naturels de ceux artificiels, la seconde à distinguer substances simples et composées; deux critères que l'auteur ne semble pas considérer 
comme pertinents ${ }^{44}$. Il s'attarde en revanche plus longuement sur le troisième, qui correspond à la différence, que nous avions notée chez Avicenne, Averroès ou Pietro d'Abano, entre les venins agissant par leur complexion et ceux le faisant par la forme spécifique. Cristoforo rappelle à cette occasion la définition que l'auteur du Conciliator donnait de la forme spécifique, le citant explicitement ${ }^{45}$. Toutefois, au moment de présenter les poisons agissant par leur seule qualité, Cristoforo adopte une position fort différente des auteurs qui l'ont précédé :

Certains venins sont quant à eux ceux qui, à ce qu'ils font par la forme, voient s'adjoindre une qualité auxiliaire manifeste, à savoir le chaud, le froid, l'humide ou le sec; les venins de cette sorte, chauds, froids, secs ou humides, diffèrent des médicaments. Les médecins ont prit l'habitude de dire que ces venins accomplissent leurs opérations par la qualité ou la complexion; cependant, il ne faut pas penser qu'ils le font par la seule qualité de leur complexion chaude, froide, etc., mais qu'ils le font par la forme [spécifique] principalement, et par ladite qualité instrumentalement ${ }^{46}$.

Pour Cristoforo, si les médecins ont pris l'habitude d'attribuer à la complexion l'action de ces venins, c'est que leurs qualités sont plus aisément sensibles, en raison de leur effet facilement identifiable : il donne ainsi l'exemple de la digestion, que les médecins attribuent traditionnellement à la chaleur naturelle, alors qu'en réalité c'est la vertu digestive qui agit, par l'intermédiaire de la chaleur qui n'a qu'un rôle instrumental ${ }^{47}$. L'auteur italien rejette donc la distinction entre poison agissant par la forme spécifique et poison agissant par la complexion. Pour lui, tous les venins, sans exception, tirent leur nature de la forme spécifique qui leur vient des étoiles, la seule division pouvant être opérée étant celle distinguant les venins dont la complexion a un rôle instrumental, et ceux pour lesquels elle ne remplit même pas ce rôle ${ }^{48}$.

21 Cette présentation nouvelle, qui nous apparaît comme l'aboutissement d'un long processus de définition et d'individualisation des poisons, a de nombreuses conséquences. Tout d'abord, pour la première fois depuis Galien, l'ambigüité de la définition du poison est résolue, Cristoforo degli Onesti le distinguant nettement $\mathrm{du}$ médicament : pour lui, le poison agit nécessairement par sa forme spécifique, « est contraire par tout son genre à la complexion humaine " et "effectue son opération principalement par la forme occulte plutôt que par une qualité manifeste $»^{49}$. Est ainsi affirmée l'autonomie des poisons, qui ne se confondent dès lors plus avec les autres substances. Un exemple donné par Cristoforo de Onestis permet de mettre en lumière l'individuation du poison en tant que substance identifiée et spécifique : il s'agit du cas de la corne de serpent. Selon une tradition ancienne, que l'on retrouve par exemple chez Avicenne, la corne de serpent (vulgairement appelée "langue de serpent »), avait le pouvoir de suinter en présence d'un venin ${ }^{50}$. L'explication généralement apportée à ce phénomène, parfois considéré avec suspicion par certains praticiens, faisait intervenir le rôle de l'air : le venin ayant la propriété de contaminer l'air environnant, ce dernier à son tour réagissait avec la corne de serpent, sur laquelle se produisait une sorte de condensation (irroratio), en raison de l'affinité de cette partie de l'animal avec la nature du venin. L'un des problèmes était bien sûr de savoir comment une telle corne pouvait avoir la propriété de réagir à tous les types de venins, même ceux d'une nature différente de celle du serpent. Grâce à sa généralisation de l'action par la forme spécifique, Cristoforo de Onestis pouvait avancer une solution simple : «l'attraction de la vapeur du venin vers cette corne se fait en raison de l'identité, ou la similitude, de la forme spécifique de la vénénosité $»^{51}$. Ainsi, les cas, déjà relevés par Avicenne ou Albert le Grand, pour lesquels la corne ne réagissait pas, s'expliquaient par le rôle de l'air 
ambiant, qui parfois ne se mêle pas à la vapeur produite par le venin: Cristoforo justifiait ainsi l'habitude prise de placer du sel sur la corne, pour que son humidité compense un air trop chaud et sec, qui empêcherait l'évaporation ${ }^{52}$. La forme spécifique $\mathrm{du}$ venin, contraire au corps humain, est donc un facteur d'unité pour tous les poisons ; elle en devient la caractéristique essentielle, les qualités liées à la complexion n'intervenant plus que dans un deuxième temps, pour expliquer les actions particulières de chaque poison.

Or, l'une des spécificités de cette forme est, nous l'avons vu, de ne pouvoir être déterminée par le raisonnement. Pour cette raison, l'action des poisons ne pouvait plus être connue, selon Cristoforo, que par l'expérience concrète : «Comme nous ignorons la quantité des éléments dans le composé, il est dès lors inévitable que nous ignorions dans les mélanges les formes spécifiques survenant selon le mélange d'une telle composition, et que nous ne puissions les connaître autrement que par l'expérience $»^{53}$. Cette primauté de l'expérience ne doit pas nous étonner en cette fin de XIV ${ }^{\mathrm{e}}$ siècle. Il a en effet été noté que, dans les derniers siècles du Moyen Âge, un intérêt toujours plus marqué se faisait jour pour les particularia et les informations tirées de la pratique médicale ou d'une expérience individuelle ${ }^{54}$. Du reste, il faut peut-être aussi y voir le rôle de la multiplication relative du nombre de crimes de poison à partir de 1300 environ $^{55}$ : la demande sociale pour une réponse savante au problème des empoisonnements a pu inciter les médecins à accentuer, de plus en plus, l'importance des empirica.

On peut bien sûr se demander quelles ont été les raisons ayant poussé Cristoforo degli Onesti à franchir ce pas décisif et à modifier ainsi la théorie toxicologique héritée des auteurs grecs et arabes. L'une des réponses possibles peut se trouver dans les autres questions posées par le professeur bolonais dans la première partie de son traité. En effet, plusieurs des problemata abordés traitent d'un sujet précis, celui des rapports entre peste et empoisonnement. Depuis la grande épidémie de 1348, la plupart des médecins avaient en effet développé une étiologie de la peste l'interprétant comme un empoisonnement de l'air se répandant parmi les êtres vivants, explication sans doute en partie à l'origine de la multiplication des traités De venenis à partir de la seconde moitié du XIV siècle $^{56}$. On ne doit donc pas s'étonner de ce que Cristoforo pose dans le sien les questions suivantes: Pourquoi un homme infecté par un air pestilentiel et vénéneux en infecte d'autres? Pourquoi dans les lieux vénéneux et pestilentiels se multiplient les animaux vénéneux comme les serpents, et dans les corps des empoisonnés les vers? Est-ce-que, enfin, les nourritures au contact de l'air pestilentiel peuvent à leur tour contaminer ceux qui les ingèrent $?^{57}$ Les réponses à ces questions, qui ne font que marginalement appel à la traditionnelle théorie des poisons pour se concentrer sur quelques conseils pour se préserver de l'infection, démontrent l'importance du lien s'étant établi entre peste et venins. Le dernier dubium est particulièrement intéressant: Cristoforo explique ainsi qu'en temps de peste, les animaux et les plantes deviennent porteurs d'une "vénénosité ", qui ne fait pas partie de leur nature propre mais peut être transmise à l'homme qui s'en nourrit, les rendant de fait vénéneux ${ }^{58}$.

La théorie de la forme spécifique permettait ainsi de mieux unifier les rapports entre venins, peste et astres: en effet, si la forme spécifique tire son origine, comme nous l'avons vu, de l'influence des étoiles sur le monde sublunaire, c'est également le cas, pour l'immense majorité des médecins, de l'épidémie de peste dont la cause est souvent 
trouvée dans une constellation particulière rendant l'air pestilentiel ${ }^{59}$. Agissant comme un venin, la peste doit donc souvent être combattue par les mêmes remèdes : il est donc logique de voir prescrits dans les deux cas thériaque, médecines cordiales voire, chez certians auteurs, diverses amulettes agissant par la forme spécifique ${ }^{60}$.

Le parcours rapide que nous avons effectué, de l'Antiquité à Cristoforo degli Onesti, a mis évidence une évolution nette: malgré les nombreuses citations d'autorité et l'apparente monotonie de la succession des traités, la théorie des poisons adoptée par les auteurs de la fin du Moyen Âge se révèle fort éloignée de celle proposée par Galien. Tout d'abord, il convient d'insister sur le fait que, peu à peu, la substance toxique a gagné son autonomie. Alors que, pour le médecin de Pergame, poisons et médicaments avaient un fonctionnement similaire, souligné par l'emploi d'une désignation unique, pharmakon, l'introduction du concept de forme spécifique a permis de mieux distinguer les premiers des seconds: désormais, le poison n'a plus grand-chose à voir avec la médecine, puisqu'il se caractérise avant tout par une forme spécifique particulière, contraire à tout le corps humain, et non par une simple disposition de sa complexion et de ses qualités. Bien sûr, il serait aisé de critiquer le recours systématique à une explication qui n'en est pas vraiment une. De fait, la forme spécifique, de par son caractère imprévisible et l'impossibilité de connaître ses causes, peut parfois sembler constituer une réponse simple à un problème complexe, et certains auteurs médiévaux ne manquèrent pas de le souligner, comme Jacques Despars qui vers 1450 l'accusait d'être "le refuge des ignorants", utilisé par ces derniers pour se sortir d'un «labyrinthe $~^{61}$. Toutefois, malgré les dangers de ces abus, l'introduction de la forme spécifique a eu pour conséquence, dans le contexte médiéval, d'encourager le recours à l'expérience, seul moyen pour connaître cette propriété : les longues listes de poisons des De venenis qui se multiplient aux $\mathrm{XIV}^{e}$ et $\mathrm{XV}^{e}$ siècles, dans lesquelles sont mentionnés de nombreuses substances et antidotes nouveaux issus d'une pratique concrète, attestent du rôle qu'a pu jouer la notion dans la revalorisation de l'expérience médicale quotidienne.

On ne saurait donc trop insister sur le fait que ce changement essentiel s'est produit au moment même où les venins faisaient l'objet d'un intérêt grandissant non seulement dans le cadre de la médecine, mais aussi dans celui de la société toute entière. Les liens entre la médecine scolastique et l'évolution plus générale de la société apparaissent ainsi particulièrement forts : loin d'être isolés et préoccupés seulement de questions théoriques abstraites, les médecins de la fin du Moyen Âge, en cherchant à toujours mieux saisir la nature réelle du poison, apparaissent guidés par les enjeux et les contraintes du monde dans lequel ils évoluent.

\section{NOTES}

1. On citera en particulier le travail de F. Collard, Le crime de poison au Moyen Âge, Paris, PUF, 2003, 308 p. Pour d'autres époques et d'autres lieux, on mentionnera F. Collard, Pouvoir et poison. Histoire d'un crime politique de l'Antiquité à nos jours, Paris, Seuil, 2007, 366 p.; F. Obringer, L'aconit et 
l'orpiment. Drogues et poisons en Chine ancienne et médiévale, Paris, Fayard, 1997, 329 p. ou J. de Maleyssie, Histoire du poison, Paris, François Bourin, 1991, 415 p.

2. On signalera L. Thorndike, An History of Magic and Experimental Science, vol. III, New-York, Columbia University Press, 1934, p. 525-45, qui reste la dernière véritable synthèse sur le sujet.

3. A. Touwaide, "Galien et la toxicologie », Aufstieg und Niedergang der Römischen Welt, 37:2, éd. W. Haase, Berlin-New York, De Gruyter, 1994, p. 1887-1986.

4. A. Touwaide, « Galien et la toxicologie », p. 1890-1895.

5. A. Touwaide, « Galien et la toxicologie », p. 1896.

6. A. Touwaide, "Stratégies thérapeutiques : les médicaments", Histoire de la pensée médicale en Occident. Antiquité et Moyen Âge, dir. M. D. Grmek, Paris, Seuil, 1995, t. 1, p. 227-237, où l'ambiguité de la notion de pharmakon est longuement mise en évidence.

7. Cf. F. Collard, «Veneficiis vel maleficiis. Réflexions sur les relations entre le crime de poison et la sorcellerie dans l'Occident médiéval », Le Moyen Âge, CIX, 2003, p. 9-57.

8. Ce mouvement d'intégration toujours plus large des phénomènes naturels dans le cadre d'une science scolastique visant à une explication globale et exhaustive du monde a déjà été plusieurs fois souligné, comme par N. Weill-Parot dans « Encadrement et dévoilement. L'occulte et le secret dans la nature chez Albert le Grand et Roger Bacon», Micrologus, XIV : Il Segreto, Florence, SISMEL, 2006, p. 151-170.

9. Pour tout ce passage, nous nous appuyons encore sur A. Touwaide, « Galien et la toxicologie », p. 1948-1972, que nous complétons par divers autres travaux, en particulier A. Touwaide, «Les poisons dans le monde antique et byzantin: introduction à une analyse systémique ", Revue d'histoire de la pharmacie, vol. 38 ( $\left.\mathrm{n}^{\circ} 290\right)$, 1991, p. 265-281.

10. En particulier dans le De simplicium medicamentorum temperamentis et facultatibus (éd. C. G. Kuhn, Claudii Galeni Opera omnia, Leipzig, 1821-1833, vol. XI, p. 601) : Sicuti ergo lactuca, si cor refrigeravit, interimit, sin concoquatur, alimentum animali efficitur, ad eundem, arbitror, modum cicuta hominem quidem celeritate distributionis interficit, sturnos vero tarditate ipsa nutrit. Si vero exiguum quiddam sumatur, mortem homini nequaquam inferet.

11. Cf. De simplicium medicamentorum temperamentis et facultatibus (éd. Kuhn XI, p. 754), signalé par A. Touwaide, "Galien et la toxicologie », p. 1970. Franck Collard, dans son Le crime de poison au Moyen Âge, p. 80, signale ainsi le cas de deux médecins savants, Raymond Chalmel et Jean de Tournemire, s'appuyant en 1380 sur l'autorité de Galien pour affirmer qu'il ne leur est pas possible de determiner si la personne décédée qui leur est présentée est morte d'empoisonnement ou d'une simple maladie.

12. Cf. De theriaca ad Pisonem (éd. Kuhn XIV, p. 281).

13. Sur la théorie toxicologique chez les auteurs arabes, on se reportera à l'article ancien, mais non remplacé, de M. Steinschneider, «Die toxicologischen Schriften der Araber bis Ende XII. Jahrhunderts : ein bibliographischer Versuch, grossentheils aus handschriftlichen Quellen», Virchows Archiv, 52.3, 1871, p. 340-375.

14. Ces textes sont présentés par D. Jacquart et $\mathrm{F}$. Micheau dans La médecine arabe et l'Occident médiéval, Paris, Maisonneuve et Larose, 1990, p. 61-74.

15. Cf. Rāzī, Liber ad Almansorem, VIII, 1, de collectionibus et aggregationibus sanationis venenorum et morsus venenosorum animalium et cautela, éd. Milan, per Leonardum Pachel et Uldericum Scinzcenceller, 1481, fol. h6 ${ }^{\mathrm{r}}-\mathrm{h} 7^{\mathrm{r}}$.

16. Avicenne, Canon, livre IV, fen 6 de venenis. Cette fen est elle-même divisée en cinq chapitres, intitulés De radicibus ejus quod scitur de dispositionibus venenorum ebibitorum et distinctione sermonis in curationibus venenorum que non sunt ex animalibus, De venenis ebibitis que sunt ex animalibus, De morsione et punctura, De morsu hominis et quadrupedum, De punctura parvorum venenosorum.

17. Sur la forme spécifique, voir B. P. Copenhaver, "Scholastic Philosophy and Renaissance Magic in the De vita of Marsilio Ficino », Renaissance Quarterly, 37, 1984, p. 523-554 ou encore N. Weill-Parot, « Encadrement et dévoilement », particulièrement p. 164sq. 
18. Avicenne, Canon, I, 2.2.1.15., de his que proveniunt ex his que comeduntur et bibuntur. Sur ce passage, voir l'introduction de M. McVaugh à Arnaud de Villeneuve, Aphorismi de gradibus, éd. M. R. McVaugh, Grenade-Barcelone, Universidad de Barcelona, 1975 (Arnaldi de Villanova Opera Medica Omnia, vol. V.1), p. 17-18.

19. Avicenne, Canon, I, 2.2.1.15. : Et sua quidem operans substantia est illud, quod forma sue speciei operatur quam acquisivit post complexionem quod quum ejus simplicia se comiscuerunt, et ex eis generata fuit res una preparavit se ad recipiendum speciem et formam additam super illud quod habent simplicia. Hec ergo forma non est qualitates prime, quas habet materia, neque est complexio que generatur ex eis, sed est perfectio quam acquisivit materia secundum aptitudinem que fuit ei acquisita ex complexione, sicut in magnete virtus attractiva (La traduction donnée est celle de D. Jacquart, La médecine médiévale dans le cadre parisien, Paris, Fayard, 1998, p. 373). Avicenne développe en plusieurs autres passages de son Canon ce concept de forme spécifique.

20. Avicenne, Canon, V, c. de qualitate compositionis: Et scias quod medicine composite conferenti ut tyriaca insunt secundum simplicia ejus vestigia et virtutes et secundum formam ipsius que non fermentatur in spatio nisi ut faciat accidere complexio ejus vestigia et virtute, et fortasse sunt meliora quam simplicium.

21. Deux passages de Galien utilisent le terme de tota substantia: le premier se trouve dans le De simplici medicina, l'autre dans le troisième livre du Tegni. Cf. M. McVaugh, « The Developpement », p. 19, n. 11.

22. Cf. Galien, De simplici medicina (éd. Kuhn, IX, p. 797) : « D’autres nous sont opposés de toute leur essence ; c'est pourquoi elles nous nuisent complètement, même si nous n'en prenons que très peu; c'est le cas de (...) toutes les autres substances qui sont délétères par leur genre et non par la quantité » (traduction d'A. Touwaide, « Galien et la toxicologie », p. 1968).

23. Avicenne, Canon, IV, 6.1.2. (éd. Venise, 1490, Hain 2207, s. gg6 $6^{\mathrm{v}}$ ) : Species venenorum sunt due, scilicet faciens operationem suam cum qualitate que est in ipso, et efficiens cum forma sua et tota substantia sua. On retrouve ici une différence de catégorie, entre la qualité et la substance, selon la définition aristotélicienne.

24. Avicenne, Canon, IV, 6.1.2. (éd. Venise, 1490, s. gg6v) : Et primum quidem aut est corrodens putrefaciens sicut lepus marinus aut inflammans calefaciens sicut euforbium aut infrigidans stupefaciens sicut opium, aut oppilans vias anhelitus in corpore sicut plumbum ustum.

25. Avicenne, Canon, IV, 6.1.2. (éd. Venise, 1490, s. gg $6^{\mathrm{v}}-g g 7^{\mathrm{r}}$ ) : Efficiens autem cum tota substantia est sicut napellus (...) et iterum de venenis sunt que agunt super unum et idem membrum sicut cantarides super vesicam et lepus marinus super pulmonem. Et de eis sunt que agunt super totum corpus sicut opium.

26. Avicenne, Canon, IV, 6.1.2. (éd. Venise, 1490, s. gg $7^{\mathrm{r}}$ ): Et si non apparent nisi casus virtutum et sudor frigidus et sincopis, tunc est de venenis que contraria sunt rebus cum tota substantia et est deterius eorum.

27. Voir en particulier Avicenne, Canon, IV, 6.1.5, medicine communes ad venena.

28. Averroès, De venenis, c. 1 (éd. Venise, 1555, s. r2v) : Omnes species mortiferorum patiuntur in duo genera, sub quibus sunt species quasi infinite. Primum est eorum, que occidunt a forma specifica (...). Genus secundum est eorum que occidunt a forma complexionata id est qualitatibus primis istorum.

29. Sur ce point, nous nous permettons de renvoyer à notre travail La réception $d u$ Canon d'Avicenne. Médecine arabe et milieu universitaire en Italie avant la Peste noire, thèse de doctorat dirigée par Danielle Jacquart, Paris, 2007. Pour la diffusion du Canon en France, voir D. Jacquart, «La réception du Canon d'Avicenne: comparaison entre Montpellier et Paris aux XIII ${ }^{\mathrm{e}}$ et XIV ${ }^{\mathrm{e}}$ siècles ", Histoire de l'école médicale de Montpellier, Actes du $110^{e}$ congrès national des sociétés savantes, Paris, 1985, p. 69-77.

30. Sur les traités des poisons en Occident à la fin du Moyen Âge, voir L. Thorndike, An History of Magic, vol. III, p. 525-45.

31. La vie de Pietro d'Abano, médecin, astrologue et philosophe mort en 1316, est étudiée dans E. Paschetto, Pietro d'Abano medico e filosofo, Florence, Nuovedizioni E. Vallecchi, 1984, 358 p. On 
pourra aussi se reporter, pour une évaluation plus générale de l'œuvre de Pietro d'Abano, à $\mathrm{F}$. Alessio, «Filosofia e scienza. Pietro da Abano», Storia della cultura veneta, vol. II : Il Trecento, Vicenza, Neri Pozza, 1976, p.171-206. Sur son De venenis, on signalera G. Sodigne-Costes, «Un traité de toxicologie médiévale : le Liber de venenis de Pietro d'Abano (traduction française du début du XV siècle) », Revue d'histoire de la pharmacie, 42 (305), 1995, p. 125-136 et F. Collard, «Le De venenis de Pietro d'Abano et sa diffusion: d'une traduction française à l'autre (1404-1593)», à paraître dans les actes du colloque international Médecine, astrologie et magie entre Moyen Âge et Renaissance : autour de Pietro d'Abano organisé à Paris, les 29 et 30 septembre 2006.

32. Pietro d'Abano, De venenis, 2 (éd. Padoue, 1473) : Tertio modo consideratur venenum quantum ad specificam differentiam quam differt species a specie, et secundum hoc dixerunt omnes medici et philosophi naturales quod venenum aliud est quod facit operationem suam a qualitate sive a complexione, et aliud quod facit hoc idem per virtutem fluentem a tota specie quod medici formam specificam vocant.

33. Pseudo-Mésué, De simplicibus, I, 1. (Cf. D. Jacquart, La médecine médiévale, p. 374).

34. Pietro d'Abano, De venenis, 2 (éd. Padoue, 1473) : Nihil aliud est nisi meritum quod unumquodque compositum ex quatuor elementis secundum majorem et minorem proportionem ipsorum elementorum in composito sortitur et meretur habere ab influentibus stellis fixis que species inferiorum compositorum respiciunt.

35. Cf. D. Jacquart, "L'influence des astres sur le corps humain chez Pietro d'Abano ", éd. B. Ribémont, Le corps et ses énigmes au Moyen Âge, Caen, Paradigme, 1993, p. 73-86.

36. Cf. L. Thorndike, An History of Magic, vol. III, p. 536.

37. Cf. L. Thorndike, An History of Magic, vol. III, p. 540 : « These medieval works on poisons have features in common, such as their dependence upon Avicenna, and repeat one another a good deal. But a fifteenth century treatise on the subject was to show a closer resemblance to the work of Christopher de Honestis than any of the fourteenth century works which we have been describing displayed to one another $»$.

38. Le traité a pour incipit: Appolinis Greci primi medicine repertoris ut longha (sic) et vigili solicitudine.

39. La présentation la plus complète de la biographie de Cristoforo degli Onesti reste encore celle de G. Sarton, Introduction to the History of Science, vol. III, Baltimore, Carnegie Institution of Washington, 1948, p. 1679-1680. Voir également T. Pesenti, Marsilio Santasofia tra corti e università. La carriera di un "monarcha medicine» del Trecento, Trévise, Antilia, 2003, p. 228-234 et, sur son commentaire au Tegni, p. 325-329.

40. Cf. G. Sarton, Introduction to the History, p. 1679 et L. Thorndike, An History of Magic, vol. III, p. 540-5.

41. Le témoin sur lequel nous nous sommes appuyés est le lat. 6910 de la Bibliothèque nationale de France. Ce manuscrit, copié au $\mathrm{XV}^{\mathrm{e}}$ siècle, comprend, outre l'ouvrage de Cristoforo degli Onesti aux fol. $87^{\mathrm{r}}-113^{\mathrm{r}}$, le neuvième livre du Liber ad Almansorem de Rāzī (fol. $1^{\mathrm{r}}-86^{\mathrm{v}}$ ), un Tractatus brevis ad conservacionem visus et recuperandum anonyme (fol. $\left.113^{\mathrm{v}}-115^{\mathrm{v}}\right)$, et enfin un recueil de consilia de Marsile de Sainte-Sophie et d'autres auteurs italiens (fol. $117^{\mathrm{r}}-144^{\mathrm{v}}$ ). Une rapide recherche dans les catalogues existants a permis de repérer au moins cinq autres témoins : le Pal. lat. 1195 de la Bibliothèque du Vatican, les Harley 3659 et Add. 30050 de la British Library, le Strozzi XV 185 de la Biblioteca Nazionale de Florence et manuscrit $n^{\circ} 110$ de la Biblioteca Comunale de Cortone. Nous remercions chaleureusement Franck Collard pour avoir attiré notre attention sur plusieurs de ces codices.

42. BNF, lat. 6910 , fol. $87^{\mathrm{r}}$ : Et eorum sique sint utilitates eligere, unde ego Christophorus de Honestis eorum documenta percurrens ad subforma problematum in scientia venenorum compilabo.

43. $\mathrm{BNF}$, lat. 6910 , fol. $90^{\mathrm{r}}$ : Expeditis communibus de venenis que secundum ordinem doctrine premittenda erant, modo veniendum est ad problemata specialia ante que premictende sunt alique dispositiones venenorum.

Cahiers de recherches médiévales, 17| 2009 
44. BNF, lat. 6910 , fol. $90^{\mathrm{r}}$ : Prima est quod quedam venenorum sunt a natura et quedam ab arte [...]. $2^{a}$ distinctio est quod tam venenorum talium a natura quam per artem quedam sunt simplicia et quedam composita.

45. BNF, lat. 6910 , fol. $90^{\mathrm{r}}: 3^{\mathrm{a}}$ distinctio quod venenorum quedam agunt per simplicem formam sine manifesto auxilio qualitatis manifeste, et hoc operatur per virtutem fluentem a tota specie quam medici specificam vocant, que "nichil aliud est, ut dicit Conciliator, nisi meritum quod unumquodque compositum ex quattuor elementis secundum majorem et minorem proportionem ellementorum in composito meretur habere ab influentibus stellis fixis que species inferioriorum compositorum respiciunt".

46. BNF, lat. 6910 , fol. $90^{\mathrm{r}-\mathrm{v}}$ : Quedam autem sunt venena que ad hoc quod a forma agunt juvantur a qualitate auxilio manifesto que qualitatis est caliditas, frigiditas, humiditas vel siccitas. Et hujusmodi venena a medicinis differunt calida, frigida, sicca vel humida. Et consueverunt medici dicere illa venena facere operationes suas a qualitate sive complexione. Nec est $/ 90^{\nu} /$ extimandum quod per solam complexionalem qualitatem caliditatem vel frigiditatem etc. ipsa operentur, sed per formam principaliter et per dictam qualitatem instrumentaliter.

47. BNF, lat. 6910 , fol. $91^{\mathrm{v}}$ : Consueverunt medici atribuere illos effectus complexioni tamquam agenti eorum magis sensibili, quemadmodum dicunt digestionem fieri a calido naturali cum tamen principaliter fiat a virtute, et instrumentaliter a calido.

48. BNF, lat. 6910 , fol. $91^{\mathrm{v}}$ : Alia vero venena que a sola specifica forma interficiunt : non faciunt hoc quia calida vel frigida, sed quia talia ut dictum est supra.

49. BNF, lat. 6910 , fol. $87^{\mathrm{r}}$ : Venenum ex toto genere contrariatur complexioni humane sic quod in parva quantitate occidit hominem. Respondeo premictens quod per venenum proprie dictum operatur principalius formam ocultam quam per qualitatem manifestam.

50. Sur l'utilisation de la langue de serpent comme préventif contre les poisons, voir F. Collard, Le crime de poison au Moyen Âge, p. 84-86. On retrouve anciennement cette affirmation dans les questions salernitaines, cf. B. Lawn, The Prose Salernitan Questions, Londres-Oxford, British Academy-Oxford University Press, 1979, questions 103 et 165.

51. BNF, lat. 6910, fol. $89^{\mathrm{r}}$ : Atractio vaporis venenosi ad dictum cornu fit ratione identitatis vel similitudinis in forma specifica venenositatis.

52. BNF, lat. 6910 , fol. $89^{\mathrm{r}}$ : Secundo infero quod non manifestatur hoc modo venenum quomodocumque presentatum, quia si prohyberetur ejus vapor aeri comiscieri, tunc non iroraretur cornu. Item si aer circumstans a cornu esset multum calidus exiccatus, non permictens vaporem infectum congelari, tunc tale signum in cornu non appareret, et ideo precipiunt sapientes quod ponatur super sale dictum cornu, ut humiditate salis reprimatur siccitas aeris circumstantis prohybentis vel impedientis evaporationem.

53. BNF, lat. 6910 , fol. $90^{\mathrm{r}}$ : Et quia ingnoramus quantitatem elementorum in composito et ideo tales formas specificas advenientes secundum mixtum talis compositionis in mixtis, necesse est nos ignorare neque de ipsis scire possumus nisi quantum docet experientia.

54. Sur ce point, voir D. Jacquart, "La scolastique médicale", Histoire de la pensée médicale en Occident. Antiquité et Moyen Âge, dir. M. Grmek, Paris, Seuil, 1995, t. 1, p. 175-210. et L. Datson et K. Park dans Wonders and the Order of Nature, 1150-1750, New York, Zone Books, 1998, 511 p. (version italienne Le meraviglie del mondo. Mostri, prodigi e fatti strani dal Medioevo all'Illuminismo, Rome, Carocci, 2000).

55. Une tentative de périodisation des crimes de poison est proposée par F. Collard dans Le crime de poison au Moyen Âge, p. 40-48.

56. Sur ce point, voir $\mathrm{N}$. Weill-Parot, «La rationalité médicale à l'épreuve de la peste : médecine, astrologie et magie (1348-1500)", Médiévales, $n^{\circ}$ 46, printemps 2004, p. 73-88, particulièrement p. 87-88. Nous avons du reste vu plus haut que le rapprochement entre peste et empoisonnement était déjà effectué par Galien. Sur la peste en général, on se reportera à J.-N. Biraben, Les Hommes et la Peste dans les pays européens et méditerranéens, 2 vol. Paris-La Haye, Mouton, 1975-1976, et F. Audouin-Rouzeau, Les chemins de la peste : le rat, la puce et l'homme, Rennes, Presses universitaires de Rennes, 2003, 371p. 
57. BNF, lat. 6910, fol. $89^{\mathrm{v}}$ (Propter quid infectus ab aere pestilentiali et venenoso inficit alios? et Propter quid est quod in locis venenatis et pestilentatis multiplicantur animalia venenosa ut serpentes et in corporibus venenatis lumbrici) et fol. $89^{\mathrm{v}}$ (Utrum si accidat permutatio in aliis vegetabilibus et nutrimentis (...) ad que omnia aer perstilentialis pervenit per contactum ista aliud coruptionis recipiant in relatione ad nos ex eis nutriendos).

58. BNF, lat. 6910 , fol. $89^{\mathrm{v}}$ : Ad primum dico quod talis venenositas communicata aliis animalibus absolute non est eis venenositas viventibus et manentibus in sua specie nec ut sic repugnat suis naturis, sed communicatur eis in relatione ad nos pro quanto ipsa nos nutrientia qualitatem receptam ab eis non repungnantem sibi absolute nobis communicant, et ideo tunc non moruntur animalia ex se licet fiant nobis malum nutrimentum.

59. On ne s'étonnera pas, pour cette raison, de voir Antonio Guainerio rédiger dans la première moitié $\mathrm{du} \mathrm{XV} \mathrm{X}^{\mathrm{e}}$ siècle un traité portant sur les deux thèmes et intitulé De peste et de venenis. Sur le rapport entre peste et venins, voir N. Weill-Parot, «La rationalité médicale à l'épreuve de la peste ", p. 75-81 et J. Arrizabalaga, «Facing the Black Death: perceptions and reactions of university medical practitioners", Practical medicine from Salerno to the Black Death, éd. L. Garcia-Ballester, R. French, J. Arrizabalaga, A. Cunningham, Cambridge, Cambridge University Press, 1994, p. 237-288.

60. Cf. N. Weill-Parot, Les "images astrologiques» au Moyen Âge et à la Renaissance. Spéculations intellectuelles et pratiques magiques (XII ${ }^{e}-X V^{e}$ siècles), Paris, Honoré Champion, 2002, 992 p., en particulier le chapitre «Poison et sceau astrologique: fondement théorique et textuel d'une association », p. 573-584.

61. Cité par D. Jacquart, La médecine médiévale, p. 463-464.

\section{RÉSUMÉS}

De nombreux traités toxicologiques, souvent simplement intitulés De venenis, furent écrits et copiés tout au long du Moyen Âge. Ils réunissent bien sûr des listes de substances, en énumérant leurs effets et leurs éventuels antidotes; mais la plupart d'entre eux propose aussi une définition théorique de ce qu'est le poison, et de ce qui le différencie du médicament. L'étude analyse donc sous cet angle plusieurs textes emblématiques, de Galien à Cristoforo degli Onesti (XIV ${ }^{\mathrm{e}}$ siècle). Ce rapide parcours démontre combien le venin est, au départ, largement confondu avec le médicament, tant du point de vue de sa nature que de ses effets. L'influence du concept de forme spécifique introduit par Avicenne entraine pourtant, chez les auteurs latins de la fin du Moyen Âge, une conscience plus nette de leurs différences, qui aboutit, à la fin du XIV siècle, à une nette distinction, qui permet aux médecins d'élaborer une classification plus expérimentale des substances.

Numerous treatises on toxicology, often simply titled simply De Venenis, were written and copied throughout the Middle Ages. These were comprised of lists of substances whose effects and possible remedies are enumerated; but the majority of the treatises also included a theoretical definition of poison that distinguished it from mere medicines. This study analyses from this perspective several archetypical texts, from Galen to Cristoforo degli Onesti (fourteenth century). This brief overview demonstrates how venom was, at first, largely confused with medicine, with respect to both its nature and effects. The influence of the concept of specific form introduced by Avicenna nonetheless led Latin authors of the late Middle Ages to a clearer understanding of the 
differences between them, which led, at the end of the fourteenth century, to a clear distinction between the two, allowing doctors to construct a more experimental classification of substances.

\section{AUTEUR}

JOËL CHANDELIER

École française de Rome. 